\title{
La simplificación en el procedimiento de servicio social ${ }^{1}$
}

Simplification in the social service procedure

\author{
Citlalli, Hernández-Ortega ${ }^{2}$ \\ https://orcid.org/0000-0002-5114-5918 \\ Claudia Iveth, González-Lozano ${ }^{3}$ \\ https://orcid.org/0000-0003-2852-9216 \\ Brenda, Montemayor-Fuentes ${ }^{4}$ \\ https://orcid.org/0000-0001-6103-2725 \\ Universidad Autónoma de Nuevo León, México
}

\section{RESUMEN}

El presente artículo es producto de una revisión bibliográfica, cuyo objetivo consistió en analizar la evolución e implementación del procedimiento administrativo y académico de la unidad de aprendizaje de servicio social. La investigación fue de carácter no experimental, de tipo transeccional, univariado. Se aplicó el instrumento a una muestra de alumnos inscritos para realizar el servicio social de agosto de 2015 a agosto de 2017, en la Facultad de Ciencias Políticas y Relaciones Internacionales de la UANL. Tras la revisión documental se encontró que; la asignatura de servicio social es una unidad de aprendizaje obligatoria de octavo semestre, de las tres licenciaturas impartidas en dicha facultad, de la Universidad Autónoma de Nuevo León. Dentro del estudio se concluye que la asignatura ha facilitado el seguimiento, procedimiento y evaluación al estudiante, al trabajo del docente y del coordinador de servicio social, así como, a las instituciones receptoras.

Palabras clave: Servicio comunitario, servicio social, siase, simplificación.

\begin{abstract}
This article is the product of a bibliographic review, whose objective was to analyze the evolution and implementation of the administrative and academic procedure of the social service learning unit. The research was non-experimental, since the variable was not manipulated. The design to carry out the research was of the transectional type, since the data collection was only carried out at a single moment in time and included only one variable. The population of interest were students enrolled for Social Service from August 2015 to August 2017, at the Faculty of Political Science and International Relations of the UANL. After the documentary review it was found that; the social service subject is a compulsory learning unit for the eighth semester, of the three degrees taught at the Faculty of Political Science and International Relations, of the Autonomous University of Nuevo León. It is concluded that the subject has facilitated the monitoring and evaluation of the student, the teacher and social service coordinator, as well as the receiving institutions
\end{abstract}

Keywords: Community service, siase, simplification, social service.

Recibido: 07 de Diciembre 2018 - Aceptado: 04 de Marzo 2019

Cómo referenciar este artículo:

Hernández-Ortega, C., González-Lozano, C. I., \& Montemayor-Fuentes, B. (2019). La simplificación en el procedimiento de servicio social . Revista Política, Globalidad y Ciudadanía,80-96. Recuperado de http:// revpoliticas.uanl.mx/index.php/RPGyC/article/view/137

\section{(i) $\odot$}

1 Artículo de investigación derivado del proyecto de investigación: La simplificación en el procedimiento de servicio social.

2 Maestra en Contaduría Pública por la Universidad Autónoma de Nuevo León, Docente de tiempo completo, Facultad de Ciencias Políticas y Relaciones Internacionales Universidad Autónoma de Nuevo León. Correo: citlalli.hernandezor@uanl.edu.mx

3 Maestra en Relaciones Internacionales por la Universidad Autónoma de Nuevo León, Docente de tiempo completo, Facultad de Ciencias Políticas y Relaciones Internacionales Universidad Autónoma de Nuevo León. Correo: claudia.gonzalezlzn@uanl.edu.mx

$4 \quad$ Maestra en Ciencias Políticas por la Universidad Autónoma de Nuevo León, Docente de tiempo completo, Facultad de Ciencias Políticas y Relaciones Internacionales Universidad Autónoma de Nuevo León. Correo: brenda.politicas@gmail.com

Revista Política, Globalidad y Ciudadanía, Vol. 5 No. 10, Julio - Diciembre 2019, Universidad Autónoma de Nuevo León, Monterrey, 


\section{1.- INTRODUCCIÓN}

El presente artículo se plantea como problema analizar y entender las dificultades que presentan los alumnos de las diferentes instituciones quienes tienen la obligación de realizar su servicio social y que por falta de implementación de un programa de apoyo influya a que diferentes alumnos se queden sin una plaza. Debido a que es bien conocido que en la nueva economía y la nueva sociedad basada en el conocimiento deben rediseñar el sistema de educación superior sólo para proporcionar las habilidades apropiadas y las habilidades para afrontar los nuevos desafíos; las universidades deben comprender la nueva posición en la que se encuentran como receptores de estudiantes internacionales y el impacto positivo que eso tiene en sus regiones y países. (García, Jiménez, Zapata. 2018).

Por lo tanto, buscando un buen sistema de educación superior enfocado en el servicio social, surge la pregunta ¿Cuáles son los factores que influyen en que los jóvenes que desean realizar su servicio social no alcancen una plaza disponible?.

El artículo plantea como hipótesis: implementar un sistema que permita que los procesos para la inscripción y realización del servicio social de los estudiantes sea más fácil, práctico y eficiente, de esa manera ayudará a que más jóvenes logren terminar el servicio y que la mínima cantidad se quede sin la plaza, con lo que se facilitará la reducción de pasos dentro del procedimiento, la eliminación del traslado físicamente de estudiantes, en el registro, seguimiento, control y certificación del servicio social; ahorrando tiempo en los proceso y dinero que antes debían invertir en traslados y en exceso de impresiones. Éste proyecto sin duda alguna trae grandes beneficios para la comunidad estudiantil integral para la administración de los servicios educativos.

El presente estudio se justifica en razón de pretender resolver la problemática que se presenta con los estudiantes de la Universidad Autónoma de Nuevo León, quienes se encuentran en proceso de culminar sus estudios y que cuentan con la responsabilidad de realizar su servicio social. Con la implementación de este nuevo sistema se desea reducir los conflictos y situaciones adversas que vive un estudiante a la hora de llevar a cabo su servicio social, y sobre todo al encontrar una plaza disponible en donde realizar su servicio.

En el presente artículo se realizará un análisis de la evolución e implementación del procedimiento administrativo y académico de la unidad de aprendizaje de servicio social. El servicio social, actualmente es una unidad de aprendizaje obligatoria de octavo semestre, de las diferentes licenciaturas impartidas en la Facultad de Ciencias Políticas y Relaciones Internacionales, de la Universidad Autónoma de Nuevo León.

Así mismo, se compartirán algunas experiencias, que contribuyeron en las mejoras durante el registro, selección de plaza, inscripción, seguimiento, evaluación y certificación del servicio social; y la implementación del sistema digitalizado que agiliza el proceso, que lleva como nombre "Sistema Integral para la Administración de los Servicios Educativos" (SIASE).

Revista Política, Globalidad y Ciudadanía, Vol. 5 No. 10, Julio - Diciembre 2019, Universidad Autónoma de Nuevo León, Monterrey, México, ISSN 2395-8448. 80-96. http://revpoliticas.uanl.mx/index.php/RPGyC/article/view/137 
Esta simplificación en el procedimiento del servicio social, es el resultado de la renovación y actualización en el área académica, administrativa, investigación, infraestructura, tecnología y de responsabilidad social de la Universidad Autónoma de Nuevo León de acuerdo con la Visión 2020 .

Para comprobar lo anterior, se analizará la experiencia de los estudiantes de la Facultad de Ciencias Políticas y Relaciones Internacionales de la UANL, específicamente en el periodo de enero de 2015 a diciembre 2017, alcanzando interesantes resultados que benefician a los estudiantes, profesores, e instituciones públicas y privadas receptoras de estudiantes de servicio social, así como la misma Universidad.

La simplificación en el procedimiento de servicio social apoyó en la facilidad y rapidez con la que hoy se maneja, así como el soporte que ha dado el sistema (SIASE) para obtener indicadores que originen áreas de oportunidad para seguir cumpliendo con los objetivos y la visión de la Universidad Autónoma de Nuevo León.

\section{2.- FUNDAMENTO TEÓRICO}

\section{Concepto de servicio social y su fundamento}

El servicio social tiene su fundamento legal, en el Artículo 52 y 53 de la Ley Reglamentaria del Artículo $5^{\circ}$ de la Constitución Política de los Estados Unidos Mexicanos, relativo al ejercicio de las profesiones; al Reglamento para la prestación del servicio social de los estudiantes de las instituciones de educación superior en la república mexicana; (Reglamento de Servicio Social, 2014).

Al Artículo 38, Capítulo VIII de la Ley de Profesiones del Estado de Nuevo León: Los servicios profesionales de índole social deberán ser prestados (Congreso de Nuevo León, 2012) por las siguientes personas:

I.- Por los estudiantes de las profesiones anotadas en el artículo 5o. de la presente Ley, y que como requisito previo a la obtención de su título profesional durante un lapso no menor de seis meses ni mayor de dos años, siempre y cuando no tengan impedimento físico o mental para cumplir con el servicio; los estudiantes o egresados de las instituciones universitarias o de educación superior existentes en la entidad, deberán prestar sus servicios en el Estado de Nuevo León; la realización de éste o de actividades que con dicho carácter pudieren corresponder, según el tipo de profesión, sea por estudiantes, pasantes o graduados, que no hubieren cursado la carrera profesional en instituciones de la Entidad, podrá autorizarse bajo circunstancias de reciprocidad y de acuerdo a los reglamentos de los comités de evaluación que para cada profesión se emitan.

II.- Por cualquier profesional cuando así le sea solicitado por el Ejecutivo del Estado a través del Departamento de Profesiones, para satisfacer el interés público, siempre que la naturaleza del servicio sea de carácter temporal y no resulte incompatible con sus actividades; 
III.- Por los Colegios Profesionales.

Artículo 58, Sección 6 del Capítulo III de la Ley de Educación del Estado:

Los estudiantes del nivel de licenciatura deberán prestar servicio social en los casos y términos señalados en la Ley y demás disposiciones reglamentarias correspondientes. En éstas, se preverá la prestación del servicio social como requisito previo para obtener el título profesional. (Congreso de Nuevo León, 2019).

El servicio social se efectuará en actividades relacionadas con la formación profesional del estudiantado y con una orientación de beneficio social. (Congreso de Nuevo León, 2019)

Artículo 4, Fracción IV de la Ley Orgánica de la Universidad; en lo referente a la prestación del servicio social, como requisito para la titulación de los estudiantes.

La función de servicio social, que comprende aquellas actividades que promueven el desarrollo socio-económico y el bienestar de la población, realizándolas en términos de docencia e investigación. (Congreso de Nuevo León, 1971).

En el ejercicio de sus funciones, la universidad se rehusará a fomentar o permitir todo aquello que atente contra la paz, la vida o la dignidad humana. (Congreso de Nuevo León, 1971)

La Universidad Autónoma de Nuevo León, a través de la Dirección de Servicio Social y Prácticas Profesionales, se da a la tarea de realizar un reglamento de servicio social, cuya última actualización es en el año 2014; en este reglamento se incluyen, las normas para la prestación, seguimiento, evaluación y certificación del servicio social.

En el reglamento de Servicio Social de la Universidad Autónoma de Nuevo León, se define como servicio social, al conjunto de actividades teórico-prácticas, de carácter temporal, que ejecuten y presten los pasantes y estudiantes en beneficio de la sociedad, el estado y la comunidad universitaria. (Reglamento de Servicio Social UANL, 2014)

Es muy importante el servicio social, considerando que el principal fin, es promover la mejora social, incrementando la sensibilidad humana, y permitiendo que el estudiante desarrolle una actitud de servicio; mediante programas que contribuyan en acciones de responsabilidad social universitaria.

Considerando la finalidad del servicio social que es promover la ética, la mejora social y la responsabilidad social de los estudiantes en la Facultad de Ciencias Políticas y Relaciones Internacionales, desde agosto de 2012 únicamente se permite que los estudiantes realicen su servicio social en instituciones públicas o asociaciones civiles de beneficencia social, cumpliendo con el artículo 10 del Reglamento de Servicio Social que manifiesta la preferencia a que el servicio social se realice en instituciones públicas.

A partir de agosto del año 2015, en la Facultad de Ciencias Políticas y Relaciones Interna-

Revista Política, Globalidad y Ciudadanía, Vol. 5 No. 10, Julio - Diciembre 2019, Universidad Autónoma de Nuevo León, Monterrey, México, ISSN 2395-8448. 80-96. http://revpoliticas.uanl.mx/index.php/RPGyC/article/view/137 
cionales, el servicio social es una unidad de aprendizaje obligatoria de octavo semestre, con un valor curricular de 16 créditos, de un total de 22 créditos. Dicha unidad de aprendizaje no cuenta con calificación numérica, sino que se evalúa como CU (Cumplió).

Cabe mencionar que el servicio social no está sujeto a oportunidades de evaluación como en las otras unidades de aprendizaje impartidas en la Universidad Autónoma de Nuevo León; por lo que en el caso de que un estudiante no logre la certificación de su servicio social, deberá volver a registrarse para cumplirlo en un periodo posterior.

Para lograr acreditar los 16 créditos de la unidad de aprendizaje de servicio social, el estudiante cubrirá 480 horas (456 horas en la organización receptora y 24 de servicio comunitario), así mismo, llevará 12 horas aula-teoría siendo estas las reuniones de retroalimentación convocadas por un profesor a su cargo y el cual brindará seguimiento al cumplimiento del estudiante.

\section{Requisitos para cursar la asignatura de servicio social}

De acuerdo con el artículo 23 del Reglamento de Servicio Social de la UANL, son requisitos para realizar el servicio social, los siguientes:

I. Estar inscrito según lo establecido en el Reglamento General sobre los Procedimientos de Admisión y Permanencia de los Estudiantes.

II. Apegarse a lo establecido en el plan de estudios correspondiente.

III. Haber cubierto al menos el $70 \%$ del total de los créditos académicos del plan de estudios.

IV. Asistir al taller de servicio social impartido por la dirección

(Congreso de Nuevo León, 2014)

El primer requisito para realizar el servicio social, es que el estudiante se encuentre inscrito de acuerdo al Reglamento General sobre los Procedimientos de Admisión y Permanencia de los Estudiantes, ante la Universidad Autónoma de nuevo León; así como haber cubierto el 70 \% del total de los créditos del plan de estudios. Por lo que el estudiante, debe estar cursando el séptimo semestre de la carrera para registrarse como candidato a cursar la unidad de aprendizaje en el semestre inmediato siguiente. (Congreso de Nuevo León, 2012).

Una vez inscrito deberá acudir a un curso, impartido por la coordinación de la facultad, y cuyo principal objetivo es explicar al estudiante el proceso administrativo y académico que deberá realizar, así como los tiempos y fechas programadas para inscribirse de manera definitiva en una de las instituciones que solicitan vacantes, las cuales previamente se registraron en el sistema SIASE y realizó de manera formal la entrega de documentos necesarios para solicitar estudiantes de servicio social en un programa. Dicha entrega se realiza ante la Dirección de Servicio Social y Prácticas Profesionales de la Universidad Autónoma de Nuevo León.

Tanto el procedimiento realizado por el estudiante, como el registro de vacantes, se encuentran en un manual realizado por la Dirección de Servicio Social y Prácticas Profesionales de la Universidad Autónoma de Nuevo León, en el cual se explica de manera detallada los pasos a

Revista Política, Globalidad y Ciudadanía, Vol. 5 No. 10, Julio - Diciembre 2019, Universidad Autónoma de Nuevo León, Monterrey, México, ISSN 2395-8448. 80-96. http://revpoliticas.uanl.mx/index.php/RPGyC/article/view/137 
seguir.

Es importante destacar, que la inscripción definitiva para el semestre Enero - Junio se realiza la primera semana del mes de mayo iniciando con su servicio social el día 1 de junio. Y la inscripción definitiva para el semestre Agosto - Diciembre es la primera semana del mes de noviembre, iniciando con su servicio social el 1 de diciembre.

\section{Procedimiento para el registro y selección de plaza de servicio social antes del año 2012.}

El procedimiento antes del año 2012, es la fecha en que se inserta el sistema para registro, inscripción, selección, seguimiento y certificación de servicio social a través del SIASE (Sistema Integral para la Administración de los Servicios Educativos).

Como hoy en día, el proceso administrativo de servicio social comenzaba con un pre registro que el estudiante realizaba en la Jefatura de Servicio Social de la facultad, el registro era de manera manual, sólo se solicitaban los datos personales y el horario deseado para realizar su servicio social.

Meses después del registro, aproximadamente en los meses de noviembre y/o mayo se citaban a los estudiantes a un curso, donde se explicaba el procedimiento y se mostraban las plazas disponibles para cada una de las carreras de facultad, así como se les explicaban las instrucciones que debían de cumplir al acudir a seleccionar la vacante en la Dirección General de Informática de la UANL, ubicada en Ciudad Universitaria en San Nicolás de los Garza, Nuevo León. El horario de las citas era en función al promedio general de las calificaciones de los semestres cursados de los estudiantes interesados.

En la Dirección General de Informática acudían los estudiantes a una sala donde existían solamente un total de 30 computadoras, por lo que se organizaban en grupos de 30 estudiantes cada 20 minutos. Ahí mismo el estudiante contaba con el acceso al sistema, seleccionaban su plaza de acuerdo a los horarios de su preferencia y se les entregaba una boleta de presentación que le serviría al estudiante para acudir a una entrevista en la institución receptora de servicio social para ponerse de acuerdo en horario, actividades, funciones, etc.

Una vez que el estudiante se encontraba de acuerdo y era aceptado, nuevamente debía acudir a la ciudad universitaria a la Dirección de Servicio Social y Prácticas Profesionales de la UANL, para notificar el estatus de la solicitud de aceptación en la institución receptora, o en su caso, notificar lo contrario.

En el caso de que el estudiante y la institución no llegaban a un acuerdo, el estudiante al acudir nuevamente a ciudad universitaria a la Dirección de Servicio Social y Prácticas Profesionales de la UANL, se le mostraban las vacantes que habían quedado disponibles y seleccionaban una a la que acudiría nuevamente a entrevistarse para llegar a un acuerdo y nuevamente regresaría a notificar su aceptación o rechazo en la misma. 
Si el estudiante fuera aceptado por la institución receptora, y se encontrara de acuerdo en realizarlo en la misma, la Dirección de Servicio Social y Prácticas Profesionales de la UANL, le entregaba el nombramiento, documento oficial de inicio de su servicio social. Dicho documento contaba con 4 hojas de colores (una amarilla para el estudiante, una azul para la facultad, una rosa para la institución donde hacían su servicio social y una verde para rectoría). El nombramiento que se le entregaba, debía de ser llevado a ser firmados y sellados por la institución receptora de Servicio Social y entregarlos ya firmados y sellados a tres instancias; la primera era dejarla en la misma institución donde realizaría su servicio social, la siguiente debía entregarla en la facultad y por último en la Dirección de Servicio Social y Prácticas Profesionales de Rectoría.

Una vez que entregaba los nombramientos, iniciaba de manera oficial su servicio social. Cada mes se debía llenar un reporte mensual de manera física en la institución misma y posteriormente el estudiante entregaba a la facultad cada mes. En dicho reporte se retroalimentaba las actividades que desempeñaba el estudiante, así como la cantidad de horas que cubría en ese mes, la cual era evaluada por parte de la institución. Era de gran importancia que el documento se encontrara firmado y sellado por Recursos Humanos de la Institución para darle la formalidad y credibilidad requerida.

En ese momento en la Facultad de Ciencias Políticas y Relaciones Internacionales de la UANL, era permitido que los estudiantes realizaran su servicio social en instituciones privadas, esto debido a que no se contaba con la cantidad de vacantes suficientes para cubrir la demanda de los estudiantes. Actualmente, por fortuna no sucede esto y solamente los estudiantes pueden realizar su servicio social en instituciones públicas o asociaciones civiles siempre y cuando demuestre ser de responsabilidad social.

Al concluir el servicio social y después de verificar que la institución haya realizado los 6 reportes mensuales en el SIASE, así como la revisión del cumplimento por parte del estudiante de un total de 480 horas en la misma, el estudiante debía solicitar a la institución una constancia de terminación en donde manifestaba el haber concluido de manera satisfactoria su servicio social, así como la fecha en la que inició y lo termino. Posteriormente, el documento era firmado, sellado y membretado por Recursos Humanos de la institución. También, el estudiante debía llevar un reporte global, el cual era llenado por la institución receptora y en él se evaluaba al estudiante en su desempeño, asistencia, puntualidad, etc.

Ambos documentos se entregaban en la Jefatura de Servicio Social de la Facultad, junto con el nombramiento y un acta de nacimiento. Una vez entregados estos documentos, la Jefatura de Servicio Social de la Facultad, elaboraba una constancia donde avalaba que el estudiante había concluido satisfactoriamente. Al término del procedimiento, el estudiante debía llevar toda la papelería original y dos copias de los 5 documentos a la Dirección de Servicio Social y Prácticas Profesionales de la UANL. Una vez entregado los documentos, el estudiante esperaba aproximadamente de 2 a 3 meses para que la carta de liberación llegue a la facultad y así concluir el procedimiento. 
Como se puede observar, era un procedimiento largo en donde el estudiante realizaba un sin número de traslados de un lugar a otro y una cantidad importante de pasos con diversos documentos.

\section{Procedimiento del servicio social en la actualidad.}

Actualmente, se ha avanzado de manera significativa tanto en la simplificación del proceso como en la agilización de los tiempos, mediante la implementación de un sistema en el cual, se encuentra un solo registro en línea y en donde el estudiante solo lleva a la Jefatura de Servicio Social de la facultad, el documento impreso del SIASE. Posteriormente, se le cita a un curso en la misma facultad con el objetivo de explicar el procedimiento administrativo y académico que debe realizar, así como los tiempos de selección de plaza, mismos que puede realizar desde la comodidad de su casa.

Este mismo sistema le ofrece al estudiante las vacantes disponibles, las cuales puede seleccionar la de su preferencia e imprimir la boleta de presentación para acudir a una entrevista en la institución seleccionada.

En caso de no llegar a un acuerdo, el mismo día puede nuevamente regresar al sistema para seleccionar otra vacante y acudir a una entrevistarse con la institución.

En caso de haber llegado a un acuerdo, solo lo notifica en el mismo sistema, para posteriormente imprimir la boleta de confirmación de plaza, en la que aparecen los datos de la institución, así como el horario y fecha de inicio de su servicio social.

El seguimiento de servicio social, tanto del estudiante como de la institución receptora, es realizado mediante reportes mensuales que cada mes llenan los estudiantes y las instituciones receptoras.

Una vez concluido el servicio social y habiendo cumplido el estudiante con al menos 456 horas en la institución, con los 6 servicios comunitarios y un total de 24 horas, haber concluido y llenado los 6 reportes mensuales entre el estudiante y la institución. El sistema le permite imprimir la constancia de terminación a la institución, misma que debe entregar a la Jefatura de Servicio Social de la facultad para que ésta, a su vez, organice los documentos y realice una constancia que avale la terminación y cumplimiento por parte del estudiante de servicio social. Hasta ese momento, el profesor sube la calificación de Cumplió (CU) en el SIASE. Tanto la carta de terminación de la institución, como la carta emitida por la Jefatura de Servicio Social, son entregadas por parte de la Coordinadora de Servicio Social de la facultad a la Dirección de Servicio Social y Prácticas Profesionales de la UANL.

\section{Herramientas de apoyo para el cumplimiento de servicio social.}

La Dirección de Servicio Social y Prácticas Profesionales de la UANL, ha realizado enormes avances en los últimos 3 años, ya que el servicio social se convierte en una unidad de aprendi- 
zaje que el estudiante debe acreditar, con carga curricular de 16 créditos. Para facilitar el cumplimiento de todas las partes involucradas, se elaboraron 3 manuales, uno para el estudiante, uno para el coordinador y uno para el profesor de la unidad de aprendizaje de servicio social.

Actualmente, ya no es necesaria la carta de liberación en físico como requisito indispensable para que el estudiante se titule de licenciatura, ahora de manera automática, el sistema le permite monitorear al departamento de Escolar si el estudiante acreditó o no la unidad de aprendizaje de servicio social para continuar con su proceso de titulación.

Los periodos en los que el estudiante realiza su servicio social también cambiaron, anteriormente eran:

1) De agosto a febrero de cada año.

2) De febrero hasta agosto.

Actualmente se ajustan los tiempos:

1) Del 1 de diciembre al 30 de mayo

2) Del 1 de junio al 30 de noviembre

Adicional al cambio en las fechas de inicio y término del servicio social y del proceso administrativo que el estudiante realiza, también existe un proceso académico que el estudiante cumple en el semestre que cursa la unidad de aprendizaje de Servicio Social. El Servicio Social, es una unidad de aprendizaje semi-presencial con una cantidad de sesiones presenciales utilizadas y con el principal objetivo de dar seguimiento al cumplimiento de las obligaciones en el sistema. Adicional a ello, una cantidad de sesiones en la plataforma NEXUS para cumplir con actividades académicas y en las cuales, el estudiante realiza la entrega de evidencias que son evaluadas en línea vía NEXUS (García, 2014) (Fig. 5.2); de este modo se obliga a que todos los que participan en el Servicio Social intervengan en el proceso de seguimiento, evaluación y acreditación del Servicio Social. (Dirección de Servicio Social y Prácticas Profesionales, 2014).

El estudiante en el semestre es evaluado con 4 evidencias y un Producto Integrador, las 4 evidencias son:

1. Expectativas en la organización receptora: el estudiante realiza un ensayo de lo que esperaba de las actividades en la institución dónde realiza el servicio social.

2. Reseña en la organización: el estudiante describe las actividades que realiza, y evalúa la importancia de éstas para la organización y para su entorno.

3. Desafíos y soluciones en el servicio social: el estudiante describe las situaciones de contingencia que ha vivido durante el desarrollo de su servicio social y debe mencionar cómo se ha enfrentado y que solución ha brindado.

4. Experiencia en el Servicio Social: el estudiante narra los acontecimientos que vivió durante los 6 meses y describe las habilidades que considera logró desarrollar y de qué manera enriqueció su formación integral. 
También debe de realizar un Producto Integrador, que es un documento que contenga la descripción de las actividades realizadas durante el servicio social y una reflexión personal de los aprendizajes obtenidos durante el lapso del cumplimiento, así como si se realizó alguna aportación adicional que haya dejado durante su estancia en la institución. (Programa Sintético de la Unidad de Aprendizaje de Servicio Social UANL, 2013).

En el Producto Integrador se incentiva al prestador de servicio social a realizar una autoevaluación de la experiencia tanto en el servicio social como en su servicio comunitario.

Así mismo para el Coordinador de Servicio Social de la facultad es una herramienta, ya que permite identificar a los estudiantes que aportaron algo adicional a las actividades que realizaba en su servicio social, es decir, quienes enriquecieron y aportaron una mejora que fue adoptada en el área donde realizó el servicio y se les invita a participar en el Premio a la Excelencia Universitaria del Servicio Social y Voluntariado que otorga la H. Consejo Universitario de la Universidad Autónoma de Nuevo León, y el que por 3 años consecutivos lo ha ganado la Facultad de Ciencias Políticas y Relaciones Internacionales.

Todos los avances anteriormente descritos, han sido el resultado del gran trabajo realizado de catedráticos y expertos en la materia; sin embargo, es muy importante no descuidar las áreas de oportunidad detectadas hasta el momento; así como mantener los avances hasta el momento logrados.

Como se ha observado en solo este periodo de 2 años, hubo un cambio radical y de contar con un proceso lleno de pasos, traslados físicos y la impresión de un sinnúmero de documentos, a simplificar el procedimiento tanto del estudiante como de las instituciones que solicitan estudiantes de Servicio Social.

Es a través del Sistema Integral para la Administración de los Servicios Educativos (SIA$\mathrm{SE}$ ), que es una plataforma de la Universidad Autónoma de Nuevo León, que se optimizan los procesos administrativos de los departamentos de la Institución (Escolar, Recursos Humanos, Finanzas, Servicio Social, entre otros) para obtener información en forma oportuna y confiable. Y del software Oracle Secure Global Desktop (SGD).

\section{3.- MÉTODO}

\section{Diseño}

El diseño metodológico utilizado en el presente estudio, fue empleando el método cualitativo, desarrollando una pregunta de investigación y una hipótesis para utilizar la recolección y análisis de los datos, así afinar las preguntas de investigación o revelar nuevas interrogantes durante el proceso de interpretación.

La principal variable que se consideró, fue a través de la pregunta de investigación: ¿Cuáles son los factores que influyen en que los jóvenes que van a realizar su servicio social no alcancen 
una plaza?

La hipótesis es considerada una hipótesis general y fue utilizada para establecer de forma previa al estudio y conceptualmente, sin cuantificar las variables. La hipótesis nació del proceso de generalización a través de ciertas observaciones que se han presentado sobre el fenómeno de estudio.

En la presente investigación se tomaron como base los datos obtenidos por los reportes del Sistema de Servicio Social, estos reportes son habilitados solamente para el Coordinador de Servicio Social de las diferentes facultades de la UANL. La investigación fue de carácter no experimental, ya que no se manipuló la variable. El diseño para realizar la investigación fue de tipo transeccional, ya que la recolección de datos sólo se realizó en un momento único en el tiempo e incluyó una sola variable. La población de interés fueron los alumnos inscritos para realizar servicio social de agosto de 2015 a agosto de 2017, en la Facultad de Ciencias Políticas y Relaciones Internacionales de la UANL. Para la codificación de datos se usó una hoja tabular de Excel, en la cual se capturó la información arrojada por los reportes y se elabora la gráfica.

Paradigma Constructivista es una herramienta que nos ayuda a afirmar que el mismo es un sustento para la investigación cualitativa; a continuación se indican las siguientes afirmaciones como aportaciones principales de este paradigma.

- La realidad se la construye socialmente desde diversas formas de percibirla.

- El saber se construye de forma social por los participantes en el proceso investigativo.

- La investigación no es ajena a los valores del investigador.

- Los resultados no pueden ser generalizados en forma ajena al contexto y el tiempo (Ramos, 2015).

Para el presente proyecto se ha decidido trabajar con estudiantes de la Universidad Autónoma de Nuevo León, próximos a culminar sus estudios para probar y medir resultados de un antes y un después de existir SIASE, ya que son los principales afectados por el problema que se presentaba por la ausencia de un programa o servicio que les simplifique los trámites y procesos para realizar su Servicio Social.

\section{Participantes}

Los participantes de este proyecto de investigación fueron los alumnos inscritos para realizar Servicio Social de agosto de 2015 a agosto de 2017, en la Facultad de Ciencias Políticas y Relaciones Internacionales de la UANL.

En la investigación cualitativa, la decisión sobre el mejor modo de obtener los datos y de quién o quiénes obtenerlos se toman en el campo, ya que los participantes del estudio nos resultan desconocidos cuando se inició y es la propia información obtenida la que va guiando el muestreo. 
Con un muestreo estratificado resultará conveniente segmentar la muestra según las variables de interés. Para ello se debe conocer la composición estratificada de la población objetivo para realizar un muestreo. Una vez calculado el tamaño de muestra apropiado, este se reparte de manera proporcional entre los distintos estratos definidos en la población.

Tiende a asegurar que la muestra represente adecuadamente a la población en función de unas variables seleccionadas. Se obtienen estimaciones más precisas y su objetivo es conseguir una muestra lo más semejante posible a la población en lo que a la o las variables estratificadoras se refiere.

\section{Instrumentos}

Siguiendo la lógica de la metodología "investigación de campo", se realiza directamente esta técnica en el medio donde es presentado el fenómeno de estudio. La observación como el primer paso de este proyecto, es una técnica que consiste en observar y analizar atentamente el fenómeno, hecho o caso, recabar información y registrarla para su posterior análisis. La observación es un elemento fundamental de todo proceso investigativo, en ella se apoya el investigador para obtener el mayor número de datos, después se tiene la entrevista, encuesta, cuestionario. Esta técnica fue elegida a raíz de los datos e información observados directamente de la realidad a través del uso de técnicas de recolección de datos (entrevistas, cuestionarios y encuestas) con el fin de dar respuesta a las situaciones o problemas que presentaban los estudiantes a la hora de registrar su servicio social.

\section{Procedimientos}

La investigación científica consta de ocho pasos:

1. Formulación del problema, siempre precisa de dos pasos: 1.-La selección de una idea o tema a investigar, en este caso es el proceso tardío, los problemas o dificultades que presentaban los alumnos e incluso los maestros en cuanto la realización del Servicio Social que se tiene que realizar por parte de los estudiantes, antes del sistema digital que actualmente facilita el proceso de inscripción y es denominado Sistema Integral para la Administración de los Servicios Educativos (SIASE). 2.-Realizar una investigación exploratoria de ese tema, básicamente es revisar todos los conflictos y los factores que influyen en ese problema y la solución que se le ha dado.

2. Identificar factores importantes, los factores que forman parte del problema, es decir, que lo describen. Los factores que están correlacionados con él y los factores que inciden en él precisando sus relaciones casuales.

3. Recopilación de la información, búsqueda de los datos que permitirán confirmar o refutar una hipótesis. El científico no debe buscar confirmar las hipótesis sino probarla. Una búsqueda indebida de confirmación de las hipótesis puede dar lugar a investigaciones contrarias a investigaciones fiables.

4. Probar la hipótesis, contrastar o comparar las hipótesis propuestas con la información real obtenida en el proceso de la recopilación de datos. Para realizar esta comparación 
es preciso someter los datos a un análisis estadístico de manera que se descartaron los resultados obtenidos al azar o a algún factor no considerado. El análisis estadístico se realiza mediante técnicas como la estadística descriptiva, prueba de hipótesis o la estadística inferencial.

5. Trabajar con la hipótesis, los resultados de la investigación se expresan mediante índices aritméticos tales como porcentajes o tasas, índices de correlación, etc. Se muestran en tablas de frecuencias, gráficos, etc. de tal manera se puede extraer una conclusión.

6. Reconsiderar la teoría, la naturaleza misma de las teorías empíricas es que pueden modificarse según los resultados de las investigaciones futuras. En este sentido, la confirmación o la refutación de una hipótesis es una contribución más en la construcción de una teoría, contribuyendo de forma general en la ciencia misma.

7. Formular nuevas preguntas, la confirmación o refutación de una hipótesis es una plataforma para plantear nuevas preguntas de investigación o mejorar, actualizar o sustituir las conclusiones obtenidas.

8. Publicación de los resultados, los resultados obtenidos son sometidos a evaluación mediante un artículo, en una publicación científica, para su evaluación por parte de la comunidad científica y reiniciar el ciclo.

\section{4.- RESULTADOS}

Los resultados del presente trabajo determinan que después de simplificar el procedimiento de servicio social, que sin duda fue un trabajo exhaustivo y metódico, en el cual intervino la colaboración de catedráticos, administrativos y expertos en el área de servicio social; y al ser implementado, se observa que disminuyo la deserción y rotación de estudiantes; así como, se incrementó la eficiencia terminal en aquellos que realizan servicio social.

En este contexto, se puede observar de una manera muy clara, un antes y un después de la simplificación del procedimiento de servicio social; por mencionar algunos de los resultados positivos de la simplificación del procedimiento se encuentra:

- Incremento en vacantes.

- Incremento en la eficiencia terminal de los estudiantes.

- Reducción de pasos dentro del procedimiento.

- Ahorro de impresiones.

- Eliminación del traslado físicamente de nuestros estudiantes en el registro, seguimiento, control y certificación del servicio social; ahorrando tiempo y dinero que antes debían invertir.

Se puede comprobar lo anterior, ya que, en el mismo sistema, existe un reporte de la cantidad de estudiantes que realizan servicio social, la fecha, sus datos personales, si cumplió con el llenado de reportes mensuales de seguimiento del estudiante y de la organización receptora, así como si el estudiante entregó papelería y concluyó el proceso.

Con los datos de ese reporte, se elaboró la gráfica 7.1 y la tabla 7.1, que muestra la cantidad 
de estudiantes que realizaron Servicio Social por periodo y la eficiencia terminal de cada uno, por lo que es claro el avance en el incremento la cantidad de Servicio Social realizadas por año, y el aumento en la eficiencia terminal de las mismas.

Gráfica 1. Estudiantes que realizaron Servicio Social, del año 2012 al año 2017

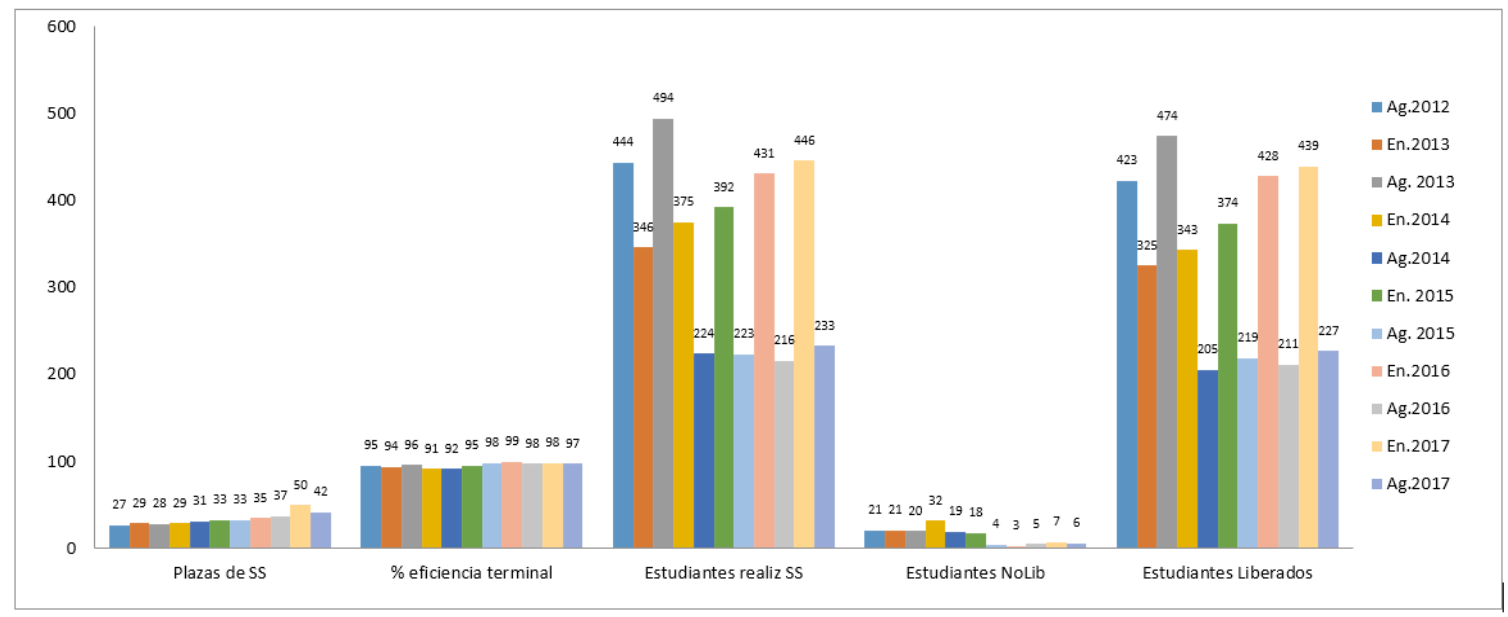

Fuente: Elaboración propia, 2018.

Tabla 1. Vacantes de Servicio Social y su eficiencia terminal, del año 2012 al año 2017

\begin{tabular}{l|c|c|c|c|c|c|c|c|c|c|c} 
& $\begin{array}{c}\text { Ago } \\
2012\end{array}$ & $\begin{array}{c}\text { Ene } \\
2013\end{array}$ & $\begin{array}{c}\text { Ago } \\
2013\end{array}$ & $\begin{array}{c}\text { Ene } \\
2014\end{array}$ & $\begin{array}{c}\text { Ago } \\
2014\end{array}$ & $\begin{array}{c}\text { Ene } \\
2015\end{array}$ & $\begin{array}{c}\text { Ago } \\
2015\end{array}$ & $\begin{array}{c}\text { Ene } \\
2016\end{array}$ & $\begin{array}{c}\text { Ago } \\
2016\end{array}$ & $\begin{array}{c}\text { Ene } \\
2017\end{array}$ & $\begin{array}{c}\text { Ago } \\
2017\end{array}$ \\
\hline \begin{tabular}{l} 
Plazas de SS \\
\hline $\begin{array}{l}\text { \% eficiencia } \\
\text { terminal }\end{array}$
\end{tabular} & 29 & 28 & 29 & 31 & 33 & 33 & 35 & 37 & 50 & 42 \\
\hline $\begin{array}{l}\text { Estudiantes } \\
\text { realizando SS }\end{array}$ & 444 & 346 & 494 & 375 & 224 & 392 & 223 & 431 & 216 & 446 & 233 \\
\hline $\begin{array}{l}\text { Estudiantes } \\
\text { No Liberados }\end{array}$ & 21 & 21 & 20 & 32 & 19 & 18 & 4 & 3 & 5 & 7 & 6 \\
\hline $\begin{array}{l}\text { Estudiantes } \\
\text { Liberados }\end{array}$ & 423 & 325 & 474 & 343 & 205 & 374 & 219 & 428 & 211 & 439 & 227
\end{tabular}

Fuente: Elaboración propia, 2018.

\section{5.- CONCLUSIONES}

Por último, se sabe que las Instituciones de Educación Superior, progresan la investigación 
junto con la docencia y la extensión, para fomentar la durabilidad del desarrollo de las comunidades y su calidad de vida; ya que en sus programas y diseños curriculares se han incluido experiencias comunitarias a través del servicio social comunitario, ayudando así a que el desarrollo humano y social de su entorno pueda tener un desarrollo visible.

En estos últimos años en los que el Servicio Social es una unidad de aprendizaje con valor curricular, donde el estudiante debe realizar un proceso administrativo y un proceso académico, para cursarla y acreditarla; es importante destacar que era de gran importancia reducir y simplificar el procedimiento de servicio social desde el registro, control, evaluación y certificación del mismo; que antes se realizaba de manera física y manual, ya que era un área de oportunidad no atendida, y que los estudiantes e instituciones requerían se atendiera. Afortunadamente hoy ya se cuenta con el Sistema Integral para la Administración de los Servicios Educativos (SIASE), donde de manera digital el estudiante, la institución receptora y los maestros, manejan ingresando al sistema desde una computadora, Tablet o celular inteligente para realizar el todo el procedimiento de servicio social.

Esta simplificación del procedimiento de Servicio Social permite contar con un control más eficiente, así como, tener mejores resultados en el seguimiento de éste. Otra de las ventajas de la simplificación es el ahorro de documentación que antes se requería imprimir y ahora solo se requiere monitorear a través del sistema. Es importante destacar que el sistema también permite evaluar el cumplimiento en lo académico de la unidad de aprendizaje al subir a NEXUS (plataforma institucional para cursos presenciales, semi-presenciales y a distancia); las cuatro evidencias y el Producto Integrador que el estudiante debe cubrir para acreditar la unidad de aprendizaje de Servicio Social.

Por lo anterior, se considera que es de suma importancia la simplificación del procedimiento de Servicio Social, ya que se toma el control desde la inscripción, el registro, la aceptación, el seguimiento, el control, el seguimiento a los Servicios Comunitarios y la liberación; sin duda aún se puede detectar más áreas de oportunidad para seguir facilitando a los estudiantes y a todos los actores que participan en el procedimiento de servicio social pero constantemente se sigue trabajando en la mejora continua del procedimiento.

Concluyendo con la idea de que actualmente las instituciones requieren de este tipo de actividades en donde logren ser pioneras de las distintitas competencias y habilidades que alcancen a desarrollar en los estudiantes principalmente aquellas actitudes de servicio con un sentido humano y con una responsabilidad social que sean capaces de desenvolverse en los diferentes contextos sociales.

Es así, como en la actualidad el servicio social debe de mantenerse en constante transformación para que los estudiantes que realizan las diferentes actividades multidisciplinarias y que van más allá de un perfeccionamiento del área profesional del prestador del servicio social, se conviertan en un motivante integrador a la realidad de los distintos panoramas del ejercicio profesional. 


\section{REFERENCIAS}

Congreso de Nuevo León, México (2012). Ley De Profesiones Del Estado De Nuevo León. Recuperado de http://www.hcnl.gob.mx/trabajo_legislativo/leyes/pdf/LEY\%20DE\%20 PROFESIONES\%20DEL\%20ESTADO\%20DE\%20NUEVO\%20LEON.pdf

Congreso de Nuevo León, México (1971). Leyes . Ley Orgánica de la Universidad Autónoma de Nuevo León. Recuperado de http:/www.hcnl.gob.mx/trabajo_legislativo/leyes/leyes/ ley_organica_de_la_universidad_autonoma_de_nuevo_leon/

Congreso de Nuevo León, México (2019). Leyes . Ley de educación del estado Recuperado de http:/www.hcnl.gob.mx/trabajo_legislativo/leyes/leyes/ley_de_educacion_del_estado/

Congreso de Nuevo León, México (2014). Leyes y reglamentos de la Universidad Autonoma de Nuevo Leon. Recuperado de https://www.uanl.mx/wp-content/uploads/2018/08/ reglamento-vigente-de-servicio-social.pdf

Diario Oficial de la Federación. (1981). Reglamento para la Prestación del Servicio Social de los Estudiantes de las Instituciones de Educación Superior en la República Mexicana. Recuperado de http:/www.normatecainterna.sep.gob.mx/work/models/normateca/ Resource/229/3/images/reglamento_prestacion_servicio_social_estudiantes.pdf

Diaz Lopez, J. C. (s.f.). Servicio Social Comunitario: Una forma de aprendizaje para los estudiantes del Área Económico-Administrativa. Recuperado de https://pdfs. semanticscholar.org/9cf7/dc27a88142f47d8c30a692378700851f2b20.pdf

Dirección de Servicio Social y Prácticas Profesionales, UANL. (2014). Manual para el prestador de Servicio Social. Recuperado de http://cidics.uanl.mx/wp-content/uploads/2018/09/ Manual-del-Prestador-de-SS.pdf

Dirección de Servicio Social y Prácticas Profesionales, UANL. (2014). Manual del profesor de Servicio Social. Recuperado de https://www.uanl.mx/wp-content/uploads/2018/08/ manual-profesor-pdf-2014.pdf

Dirección de Estudios de Licenciatura. (2011). Modelo Académico de Licenciatura. Aprobado por el H. Consejo Universitario el 23 de marzo de 2011. México: Autor. Escalante Araiza, M. D. (2018). Rescatar el servicio social universitario: un estudio al noroeste de México. Recuperado de https://www.researchgate.net/publication/329799286_Rescatar_el_ servicio_social_universitario_un_estudio_al_noroeste_de_Mexico

García Waldman, D. H., Jiménez Quintana, P. y Zapata Morán M. G. (2018). La Paradiplomacia Universitaria: La internacionalización de la educación superior en América. Revista Política Globabilidad y Ciudadanía. Vol 4. Num 8. pp.37-47. Recuperado de http://revpoliticas. uanl.mx/index.php/RPGyC/article/view/101

Ramos, C. (2015). LOS PARADIGMAS DE LAINVESTIGACIÓN CIENTÍFICA. Recuperado de http://www.unife.edu.pe/publicaciones/revistas/psicologia/2015_1/Carlos_Ramos.pdf 
96

Universidad Autonoma de Nuevo León (2014). Leyes y Reglamentos de la Universidad Autónoma de Nuevo León. Reglamento de Servicio Social. Recuperado de https://www. uanl.mx/wp-content/uploads/2018/08/reglamento-vigente-de-servicio-social.pdf

Universidad Autonoma de Nuevo León (s.f.). Servicio Social. Recuperado de https://www. uanl.mx/tramites/servicio-social/ 